\title{
Recherche et politiques publiques environnementales: vers un modèle d'interactions ${ }^{\star}$
}

\author{
Corinne Larrue*
}

Professeure en Urbanisme et Aménagement, École d'urbanisme de Paris, Lab’Urba, Université Paris-Est Créteil, Marne la Vallée, France

L'appui de la recherche aux politiques publiques environnementales est soutenu au niveau national depuis de nombreuses années par le ministère français de l'Environnement. Cette implication des instances politico-administratives dans les processus d'incitation à la recherche a pris une place à part dans l'échiquier des acteurs de l'incitation à la recherche. À côté des recherches dites fondamentales, favorisées par les instances de gestion de la recherche type CNRS ou l'Agence nationale de la recherche (ANR), le ministère de l'Environnement entend développer une recherche plus finalisée, dont les objectifs assignés sont de soutenir de manière plus ou moins directe l'élaboration ou la mise en œuvre des politiques publiques environnementales. Il s'agit d'aider à la conception de mesures à mettre en place, de préciser les objectifs écologiques à retenir ou d'améliorer la compréhension des processus sociaux induits par la mise en œuvre des politiques publiques à l'échelle de différents territoires. Ces recherches dites finalisées sont conçues par les instances politico-administratives comme nécessairement pluridisciplinaires, associant notamment sciences de la nature et sciences sociales. Elles doivent également impliquer de manière plus ou moins formalisée les acteurs concernés sur le terrain. Enfin les résultats de ces travaux doivent être mis en visibilité et rendus accessibles aux sphères politico-administratives.

Après une trentaine d'années d'expérience, il est possible - et il vaut la peine - de caractériser l'émergence du «modèle» d'association entre recherche et politiques publiques qui s'est ainsi construit progressivement et d'en identifier les effets tant pour les acteurs politico-administratifs que pour la communauté des chercheurs impliqués dans ces processus. Cette réflexion s'appuiera d'une façon générale sur mon expérience de

\footnotetext{
^ Voir dans ce numéro, le texte d'introduction de Marcel Jollivet.

*Auteur correspondant : corinne.larrue@u-pec.fr
}

chercheuse en «sciences sociales de l'environnement» depuis le début des années 1980 et, plus particulièrement, sur celle de responsable scientifique d'un programme de recherche financé par le ministère de l'Environnement et le plan Urbanisme construction architecture (PUCA) du ministère de l'Équipement durant cinq années: le programme Politiques territoriales et développement durable (2003-2008).

Ce propos sera structuré en deux principaux points : d'une part, une mise en perspective historique des interactions entre le ministère de l'Environnement et la communauté des chercheurs; d'autre part, une réflexion sur les dispositifs en place et leurs résultats en termes de percolation des problématiques d'action publique dans les milieux de recherche et de mobilisation des travaux de recherches dans les sphères décisionnelles. En conclusion, quelques éléments de prospective seront proposés.

\section{Les interactions entre actions publiques environnementales françaises et communautés des chercheurs : un aperçu historique}

Plusieurs jalons peuvent être proposés pour retracer ces interactions dans le temps et les qualifier.

On peut tout d'abord rappeler, comme le faisait JeanPatrick Leduc, ancien directeur du Muséum d'histoire naturelle lors du colloque consacré en 2011 aux 40 ans du ministère de l'Environnement $^{1}$, que l'action publique que l'on peut qualifier d'environnementale avant l'heure s'est constamment appuyée sur les travaux des

\footnotetext{
${ }^{1}$ Le Duc J.-P., 2013. Le rôle des chercheurs et des scientifiques, Pour Mémoire, Hors Série, 18-24, https://ecologique-solidaire. gouv.fr/sites/default/files/Pour mémoire $n^{\circ}$ hors série 40 ans ministère environnement printemps 2013 .pdf.
} 
chercheurs et a toujours bénéficié de leur implication. Il va même plus loin en présentant la création du ministère comme « un acte politique qui a reconnu une préoccupation de la société portée par ces scientifiques». Le panorama qu'il dresse du rôle des chercheurs et des scientifiques dans l'histoire de la création du ministère dans une perspective du long terme (depuis $\mathrm{XVII}^{\mathrm{e}}$ siècle) met ainsi en relief la création des jardins du roi, du Muséum d'histoire naturelle, de la Société nationale d'acclimatation ${ }^{2}$ mais aussi des publications scientifiques comme autant de jalons majeurs de cette montée en puissance des questions environnementales avant même que l'action publique ne s'en empare.

Plusieurs auteurs sont des marqueurs de l'inscription de ces préoccupations dans l'espace public. On pense, en France, à Jean Dorst qui a eu un rôle important pour attirer l'attention sur la question de la nature et sur les atteintes qu'elle subit ${ }^{3}$; aux États-Unis, à Rachel Carlson ${ }^{4}$. En France, la mise à l'agenda politique des questions environnementales doit également beaucoup aux travaux de prospective développés par Bertrand de Jouvenel ${ }^{5}$.

C'est tout d'abord à travers la production d'expertises, nécessaires pour élaborer ou mettre en œuvre les politiques environnementales qu'ont été mobilisés les milieux de la recherche. La mise en place, dans les années 1980, à l'échelle du territoire français, des Zones naturelles d'intérêt faunistique et floristique (ZNIEFF) constitue un exemple particulièrement révélateur de l'importance de ces interactions entre le ministère de l'Environnement et - en l'occurrence - les sciences écologiques. L'identification de ces zones s'est appuyée sur la mobilisation des chercheurs de terrain qui seuls avaient une connaissance localisée des espaces potentiellement intéressants à préserver. Et c'est bien sur la base de cette connaissance scientifique que des priorités d'action ont été définies.

Mais ces interactions n'en sont pas restées là. Très rapidement après sa création, le ministère de l'Environnement a été conduit à combler les lacunes dans les connaissances qui lui étaient nécessaires via l'orientation de la recherche. La mobilisation des chercheurs au travers du Service des études et de la recherche créé à l'instigation de Serge Antoine ${ }^{6}$ devait permettre d'as-

\footnotetext{
${ }^{2}$ Devenue Société nationale de protection de la nature, puis Fédération française des sociétés de protection de la nature (FFSPN), puis finalement France nature environnement (FNE).

${ }^{3}$ Cf. notamment Dorst J., 1965. Avant que Nature meure, Paris, Delachaux et Niestlé.

${ }^{4}$ Carson R., 1962. The silent Spring, Boston, Houghton Mifflin.

${ }^{5}$ De Jouvenel B., 1969. Arcadie, essai sur le mieux vivre, Paris, SEDES

${ }^{6}$ Devenu Service de la recherche, du traitement de l'information en environnement (SRETIE) au début des années 1980.
}

seoir progressivement la légitimité de l'action publique environnementale (qui avait du mal à se faire entendre). Ce souci de répondre à des enjeux sociétaux et d'action publique de la manière la plus étayée possible, et donc sur la base d'une expertise étendue, est à l'origine des programmes de recherche finalisée que le ministère a pu porter jusqu'à il y a peu.

Ces programmes ont ainsi été amenés à jouer un rôle particulièrement important dans la construction d'un milieu scientifique dans le champ de l'environnement. Car si l'on peut dire que le ministère de l'Environnement et plus généralement l'action publique environnementale ont beaucoup bénéficié des chercheurs, on peut également avancer que l'inverse est aussi vrai: la montée en puissance de la recherche environnementale française doit beaucoup au ministère de l'Environnement. Il n'a pas seulement fourni un soutien financier aux chercheurs: il a été une réelle source d'incitation à l'interdisciplinarité tout en leur permettant de gagner en légitimité dans leur propre discipline.

Ces programmes ont en outre trouvé un appui auprès des agences qui gravitaient autour du ministère. Que ce soit du fait des Agences de l'eau ou de l'ADEME à partir de 1991 (ou des agences précurseurs de l'ADEME antérieurement) un certain nombre de travaux de recherches et d'études appliquées ont été réalisés dans le cadre de ces programmes de recherche en réponse à des commandes publiques.

Cela a été le cas notamment dans le domaine des sols et des déchets (Programme sols et déchets), puis de la pollution de l'air (programme PRIMEQUAL), puis des risques (programme RIO), des conflits et de la concertation (programme Concertation décision environnement, $\mathrm{CDE}$ ), de l'eau (programme Eau et territoire), de l'agriculture (programme DIVA), etc. Progressivement, un modus operandi s'est constitué au sein de ces programmes, à l'instar de ce qui a été mis en place dans le cadre du programme - précurseurPRIMEQUAL : constitution d'un Comité d'orientation, regroupant les parties prenantes et d'un conseil scientifique regroupant des représentants des différentes familles de chercheurs concernés, lancements d'appels d'offres, animation et suivi via des séminaires faisant débattre chercheurs et praticiens... Cette organisation permettait au ministère de donner une certaine légitimité à son action de pilotage de la recherche, et aux communautés de chercheurs, d'en trouver une dans un domaine de recherche, celui de l'environnement, qui n'était pas particulièrement porteur jusque dans les années 1990.

D'autres organismes de pilotage de la recherche se sont également mobilisés : notamment le CNRS avec la création du Programme interdisciplinaire de recherche sur l'environnement (PIREN), en 1979; puis le niveau 
européen notamment à partir du $3^{\mathrm{e}}$ Programme cadre de recherche et développement, PCRD; et enfin l'Agence nationale de la recherche.

Mais la spécificité de l'action du ministère de l'Environnement est liée à cette volonté de créer et d'animer des communautés de chercheurs via des séminaires et colloques au sein desquels étaient débattus les travaux en train de se faire avec les parties prenantes représentées dans le comité d'orientation. Cela témoigne de l'existence d'une volonté explicite de faire un lien entre les résultats de recherche et leur appropriation par un certain nombre d'acteurs. Cette dynamique de mise en débat des recherches est moins présente dans d'autres organismes de recherche comme le CNRS ou l'ANR, où l'on reste plus sur un format de débat entre pairs. Cette proximité établie entre acteurs de l'action publique environnementale et chercheurs a également permis de faire évoluer les questionnements tant d'un côté que de l'autre.

\section{Le programme «Politiques territoriales et développement durable »: un retour d'expérience}

Ce programme ${ }^{7}$ est une bonne illustration des interactions entre action publique environnementale et recherche telles qu'elles se sont établies dans les années 2000. Il est clos depuis une petite dizaine d'années (il a existé de 2003 à 2008), ce qui permet un retour d'expérience pour apprécier en quoi il fut innovant et ce que furent ses limites.

La thématique retenue pour ce programme l'inscrivait résolument dans cette perspective de recherches finalisées : son intitulé mettait en scène d'emblée la question des acteurs, leur capacité à se saisir de ce nouvel objet : le développement durable, à leur échelle. D'ailleurs, le premier appel d'offres lancé en 2003 mettait bien en exergue cet objectif: «ce programme cherche à traduire les attentes des acteurs territoriaux et à leur apporter des éléments de réponse aux tensions qu'ils rencontrent entre des enjeux globaux (changement climatique et effet de serre, lutte contre les inégalités sociales économiques et écologiques, etc.) et des nécessités de développement local (économique, de mobilité, habitat, etc.) et à la manière de concilier dans leurs stratégies de développement les exigences, parfois contradictoires, du court et du

\footnotetext{
${ }^{7}$ Répertorié sous le sigle D2RT (Développement durable recherche territoire), ce programme procédait d'une initiative du ministère de l'Environnement et du Plan urbain, construction architecture, PUCA. Les financements étaient assurés par chacun des deux partenaires sur la base d'une évaluation scientifique conjointe. J'en ai présidé le conseil scientifique de 2003 à 2008.
}

long terme.». De manière plus subliminale, l'objectif du programme était de développer et capitaliser les connaissances qui permettraient aux décideurs de rendre plus cohérentes leurs politiques territoriales. Pour les acteurs environnementaux, notamment à l'échelle centrale, le développement durable apparaissait comme une opportunité pour dépasser les difficultés récurrentes du cloisonnement des disciplines et des métiers.

Deux appels à projet ont été lancés, l'un en 2003 qui a permis de financer 25 projets et l'autre en 2005 qui a permis d'en financer 21 autres. Quatre grands axes structuraient ces appels :

- axe $1:$ l'évaluation des politiques publiques au regard du développement durable;

- axe $2: 1$ 'articulation spatiale, thématique et temporelle des politiques publiques;

- axe 3: territoires et modes de gouvernance;

- axe 4 : les «inégalités écologiques».

L'axe 3 a été particulièrement investi par les chercheurs lors du premier appel d'offres, conduisant à restreindre l'appel à projet de 2005 aux trois autres axes. À noter que la question des inégalités écologiques n'a réellement été investie par les chercheurs que lors de ce dernier appel.

Ces axes de développement des recherches à mener avaient été préalablement identifiés lors de travaux préliminaires. Le programme D2RT s'inscrit en effet dans la suite du programme intitulé «Ville et écologie» mené en partenariat entre le PUCA et le ministère de l'Environnement de 1992 à 1999. Sa mise en place a été nourrie par des ateliers organisés conjointement par ces deux institutions, mobilisant un grand nombre d'acteurs ainsi que des chercheurs autour des questions de développement durable dans leur dimension spatiale. Ces ateliers ont été principalement organisés par le PUCA de 2001 à 2003 mais en association avec le ministère de l'Environnement. En outre, un certain nombre d'études ont été réalisées en préparation à la mise en place du programme de recherche: études de benchmark $^{8}$ sur les recherches menées à l'étranger sur cette thématique; recensement et analyse des attentes en provenance des acteurs territoriaux et des bureaux d'étude en aménagement et urbanisme; enquête et analyse des besoins des services de l'État.

On le voit, le programme mis en place n'est pas né de nulle part: il s'inscrit à la fois dans un processus long de coopération interministérielle, et s'appuie sur des réflexions mêlant à la fois des chercheurs et des acteurs du niveau central comme du niveau territorial.

\footnotetext{
${ }^{8}$ Il s'agit d'une étude bibliographique menée par la Fondation des villes sur les publications parues sur ce thème.
} 
La formalisation des appels à projets de recherche a été assurée par le conseil scientifique mis en place en 2003.

À noter que ces travaux préparatoires ont été réalisés sans liens avec la démarche menée parallèlement par le CNRS pour l'élaboration d'un programme de recherche sur cette même thématique du développement durable (plus spécifiquement urbain pour le CNRS). On rappellera la séparation assez claire entre les deux programmes qui allait être lancés de manière quasi concomitante: l'un celui du CNRS (intitulé «Développement urbain durable») annonçant dès le départ une posture très critique du développement durable et une volonté de rester dans un entre soi de chercheurs; l'autre celui porté par le ministère et le PUCA, volontairement plus en lien avec l'action publique, et cherchant à confronter et débattre des positions des chercheurs et des acteurs publics ${ }^{9}$.

L'organisation du programme D2RT n'a pas dérogé à la structure évoquée ci-dessus : pilotage dual via un comité d'orientation regroupant des représentants des directions des deux ministères responsables et de leurs établissements publics, ainsi que des délégués (ou membres) d'autres ministères et d'autres groupes d'intérêts: associations, élus, professionnels ${ }^{10}$ (ce conseil s'est réuni trois fois); et un conseil scientifique, composé de treize membres, mobilisant les disciplines suivantes: sciences politiques, géographie, urbanisme et aménagement de l'espace, écologie, économie (ce conseil s'est réuni onze fois). En outre, neuf séminaires et trois colloques d'envergure ont été organisés au titre de l'animation du programme. Ils ont rassemblé les chercheurs qui bénéficiaient d'un financement de leurs projets, mais également des acteurs territoriaux, des agences d'urbanisme, des bureaux d'études et des représentants des ministères de l'Équipement, de l'Environnement ou de l'Agriculture. Ce n'était pas forcément les mêmes personnes qui participaient à chaque séminaire ou lors des colloques, mais la présence de ce type de représentants était constante.

\footnotetext{
${ }^{9}$ Cette dichotomie s'est atténuée par la suite, puisque le Programme interdisciplinaire de recherche ville et environnement (PIRVE) est en partie la suite de D2RT. Certains des membres du CS de D2RT figurent également dans le Conseil Scientifique du PIRVE, assurant ainsi une sorte de continuité ou de transmission des acquis, d'un programme à l'autre.

${ }^{10}$ Le Comité d'orientation était composé de 28 membres issus d'organismes ou institutions diverses, notamment: l'ensemble des ministères, la Délégation ministérielle à la ville, les directions régionales de l'équipement, la DATAR, l'ADEME, l'Association française des conseils des communes et régions d'Europe, l'Association des maires pour l'environnement et le développement durable, la Fédération nationale des agences d'urbanisme, la Fédération des parcs naturels régionaux de France.
}

Que tirer de cette expérience au regard de la construction d'un modèle de coopération acteurs/chercheurs? Dans quelle mesure les problématiques au centre du programme ont-elles été investies par les chercheurs? Dans quelle mesure les résultats produits par les chercheurs ont été appropriés par les acteurs environnementaux centraux et territoriaux?

\section{En ce qui concerne l'appropriation des thématiques par les chercheurs}

Ce programme a donné lieu à une réelle mobilisation de la communauté scientifique sur la problématique de la dimension territoriale du développement durable; ce qui n'était pas gagné a priori. Le conseil scientifique a été un véritable espace de débats, de circulation des idées, de suivi des chercheurs, de montée en généralité et de validation scientifique, des travaux qui étaient menés. Les membres du conseil scientifique participaient régulièrement aux rencontres du programme (séminaires et colloques) et y jouaient un véritable rôle de cheville ouvrière. Cet investissement du conseil scientifique a été lourd pour ses membres, sans doute plus que le bénéfice intellectuel et de mise en réseau qu'ils ont pu en tirer.

Le programme a ainsi permis de donner une certaine légitimité à travailler sur cette problématique. Il a permis de démontrer que le développement durable pouvait être un objet de questionnement scientifique à part entière. Les retombées plus classiques en termes de publication se sont étalées jusqu'à 5 ans après la fin du programme.

À noter toutefois, que la thématique du programme, par sa nouveauté pour les milieux de la recherche française à cette époque, a conduit à une mobilisation assez importante de jeunes chercheurs. Par ailleurs, les critiques émises par le conseil scientifique sur la qualité des rapports finaux des projets, rendent compte de travaux encore exploratoires, liés à des questions assez difficiles, explorant des champs nouveaux, et manquant globalement d'analyse. Le bilan tiré à l'issue du programme pointait qu'à l'échelle du programme, on n'avait pas fait de saut global sur l'idée de «territoires durables »; peu de travaux conceptualisaient ces questions. Mais qu'en revanche on avait progressé sur des fronts spécifiques, notamment sur celui des inégalités écologiques.

Enfin, d'un bilan plus précis du déroulé et des retombées de l'ensemble du programme qui a été réalisé à sa clôture ${ }^{11}$, il ressort un résultat particulièrement

\footnotetext{
${ }^{11}$ Notamment dans le cadre d'un séminaire associant le conseil scientifique et les chargés de mission du programme au sein des deux organismes financeurs, et d'autres chercheurs. Des enquêtes ont également été conduites dans ce cadre.
} 
intéressant, qui n'avait pas été anticipé en tant que tel: celui de l'articulation des travaux de recherche avec les formations dans le champ de l'environnement et de l'aménagement. La plupart des recherches menées ont en effet donné lieu à implication d'étudiants, de stagiaires, et ont été diffusées dans le cadre de ces formations. Cette articulation est d'autant plus intéressante qu'il s'agit de la formation des acteurs de demain.

\section{En ce qui concerne l'appropriation des résultats de la recherche par les acteurs}

Le comité d'orientation est en principe l'une des instances clef pour s'assurer du dialogue entre acteurs et chercheurs. Mais la lourdeur de sa composition et la faiblesse du nombre des réunions ont fait qu'il a souffert d'un manque d'assiduité. Il semble aussi que les préoccupations des services des ministères étaient trop ancrées sur des questions de court terme pour que les personnes chargées de les représenter s'intéressent de près aux travaux menés ${ }^{12}$. Le comité d'orientation est resté de ce fait assez éloigné de la vie du programme. Il n'était pas le lieu d'une réflexion partagée avec les chercheurs. Seule, la présidente du conseil scientifique y était présente (pour exposer les projets de recherche sélectionnés et les résultats qu'on pouvait en attendre). Il s'est borné à donner la priorité à certains projets parmi des projets considérés comme scientifiquement bons.

Il a néanmoins permis une certaine mise en visibilité du programme auprès des différents acteurs. On a pu constater au fil du déroulé du programme une attention portée tant par les chercheurs que par les acteurs présents aux débats autour des projets en train de se faire. Ces débats mettaient en exergue les décalages entre les préoccupations des acteurs et les préoccupations des chercheurs, et les différences des temporalités dans lesquelles les uns et les autres inscrivent leur action. Ces dialogues ont également été mis en place à l'échelle des projets : pratiquement toutes les recherches ont proposé un terrain d'application (ce qui était ffortement conseillé dans le texte des Appels à proposition de recherche). Le degré d'interaction avec les acteurs des territoires pouvait être très important ${ }^{13}$. Au niveau des acteurs de terrain, il a donné lieu à une appropriation silencieuse progressive sur la scène même de l'action.

\footnotetext{
${ }^{12} \grave{A}$ noter toutefois que, n'ayant pas suivi les relations développées en interne entre les chargés de mission en charge du suivi du programme de recherche au sein du ministère de l'Environnement et du PUCA, et leurs hiérarchies ou autres instances assurant la connexion entre le programme et les activités des ministères concernés, je n'ai donc qu'une vision partielle.

${ }^{13}$ Comme dans le cas du travail mené à Aix-en-Provence, par l'équipe de l'université.
}

$\mathrm{Au}$ total, sur la base de cette expérience, on peut avancer que l'articulation entre les chercheurs mobilisés dans le cadre du programme et les représentants de l'action environnementale s'est faite de manière assez diffuse, par percolation, par acclimatation progressive.

\section{Une organisation à améliorer}

Le bilan tiré du programme a montré l'insuffisance de références produites au regard des demandes explicites des acteurs territoriaux. Malgré les efforts déployés pour la développer, la connexion des recherches avec l'action environnementale est demeurée assez faible. Même si ce programme a bien joué un rôle d'interface entre action publique et recherche, sa capacité à produire des réflexions mobilisables dans le cadre de l'action est restée très en deçà des attentes.

Toutefois, on notera qu'un groupe de travail réunissant acteurs (représentants des ministères de l'Environnement et de l'Équipement, représentants de collectivités territoriales) et chercheurs (notamment parmi ceux impliqués dans le programme) a été mis en place dans le cadre du programme en 2007, organisé par les deux organismes financeurs. Ce groupe, qui ne s'est réuni que deux fois avait pour objectif, sur la base de bilans d'expériences de coopérations réussies (ou non) entre chercheurs et acteurs ou de valorisations réussies en direction des acteurs, d'identifier les modalités de collaborations entre acteurs et chercheurs les plus à même de produire de meilleures recherches en direction des politiques publiques et de valoriser plus efficacement les travaux de recherche existants pour venir en aide aux décideurs. Si cette expérience n'a pas vraiment eu de suite, elle a néanmoins permis de reconsidérer la place des acteurs dans les programmes qui doivent venir en appui aux politiques publiques. À cet égard l'idée d'expérimenter une valorisation de recherches vers les territoires devait être explorée ${ }^{14}$.

De ce rapide panorama, il ressort que les programmes dont il a été question ont largement contribué à mettre les problématiques environnementales à l'agenda de la recherche. Les chercheurs qui s'y sont engagés ont joué le rôle pionnier que l'on attendait d'eux. Les communautés de chercheurs qui se sont créées depuis une cinquantaine d'années ont forcé les barrages pour permettre à tout un ensemble de problématiques de voir le jour et d'être abordées en confrontant le point de vue de la recherche et le point de vue de l'action.

On l'a vu également, les interactions entre acteurs et chercheurs ne sont ni naturelles ni faciles. Il y a une réelle nécessité à mettre en place une organisation pour ce faire,

\footnotetext{
${ }^{14}$ Ici encore, ayant quitté mes fonctions de présidente du conseil scientifique en 2008, je n'ai pas suivi ce prolongement.
} 
non seulement à l'échelle nationale mais également à l'échelle des projets. Cette organisation doit non seulement être pensée en amont, mais considérée en tant que telle et bénéficier d'un temps spécifique consacré à ces interactions (un travail dont les retombées ne sont pas toujours valorisables ni par les chercheurs ni par les acteurs). Ce temps est nécessaire pour que s'ajustent les connaissances et les besoins de connaissances respectifs des chercheurs et des acteurs. La recherche industrielle en fournit un bon exemple en associant recherche et développement.

Notons également les limites temporelles d'une autre nature de toute recherche finalisée. Ainsi la problématique politique du développement durable est maintenant dépassée. On est passé à d'autres thématiques, celle de la transition, de la résilience. Ce qui montre que ce type de programme associé au temps de l'action est aussi borné par ce même temps de l'action.

La période dont il vient d'être question est par certains côtés une période enviable. Aujourd'hui, en effet, l'aide à la conception de l'action publique devient plus difficile à mettre en place tant les systèmes d'action sont contraints et rendus rigides. Les exigences qui en résultent rendent de plus en plus difficile aux acteurs d'entendre la parole du chercheur. L'impossibilité dans laquelle ils sont de prendre en compte directement les résultats des recherches conduit à des dialogues de sourds.

S'il est une conclusion que l'on peut tirer de ce rapide survol, c'est bien de porter une attention toute particulière à cette question et de s'interroger sur les façons d'y répondre. Elle doit être totalement associée à l'attention à porter à la pluridisciplinarité, qui constitue l'autre volet majeur d'une recherche finalisée. Elle est aussi inséparable de ce qui est l'objectif final de cette démarche de recherche: celui, au-delà de l'appui à l'action publique, d'être un support pour un débat plus large associant non seulement les acteurs des politiques publiques mais l'ensemble des citoyens, qu'ils soient organisés ou agissant à titre individuel.

Citation de l'article : Larrue C., 2017. Recherche et politiques publiques environnementales : vers un modèle d'interactions. Nat. Sci. Soc. 25, S12-S17. 